Revue d'histoire de l'Amérique française

REVUE D.HISTOIRE DE L'AMÉRIQUE FRANÇAISE

\title{
La Canadienne pendant les troubles de 1837-1838
}

\section{Marcelle Reeves-Morache}

Volume 5, numéro 1, juin 1951

URI : https://id.erudit.org/iderudit/801686ar

DOI : https://doi.org/10.7202/801686ar

Aller au sommaire du numéro

Éditeur(s)

Institut d'histoire de l'Amérique française

ISSN

0035-2357 (imprimé)

1492-1383 (numérique)

Découvrir la revue

Citer cet article

Reeves-Morache, M. (1951). La Canadienne pendant les troubles de 1837-1838.

Revue d'histoire de l'Amérique française, 5(1), 99-117.

https://doi.org/10.7202/801686ar d'utilisation que vous pouvez consulter en ligne.

https://apropos.erudit.org/fr/usagers/politique-dutilisation/ 


\section{LA CANADIENNE PENDANT LES TROUBLES DE $1837-1838$}

Je souhaiterais faire voir qu'à une époque douloureuse de notre histoire, nos bisaïeules avec les humbles moyens à leur disposition, ont cherché à bien servir. Leur action a été le plus souvent sans éclat; peut-être ont-elles fait autant que personne pour la libération nationale. Nous connaissons assez bien le comportement des hommes, de ceux qu'on a appelés les "Patriotes". A lire nos Histoires, il semblerait que la femme a traversé sans qu'il lui en coate, la terrible tourmente. Je dédie ces quelques notes à ceux que tout un chapitre de notre passé pourrait tenter. Et je les transcris simplement à la file, sous cette triple rubrique:

Les femmes ont collaboré activement à l'insurrection.

Elles ont souffert.

Elles ont console.

\section{I - ElLES ONT COLLABORÉ}

A l'été de 1837, les femmes de Saint-Benoît, des Deux-Montagnes, attirent particulièrement l'attention. Le 13 aout, à une assemblée du Comité central et permanent ${ }^{1}$, le secrétaire communique aux membres une pétition de Madame Girouard, épouse de Jean-Jacques Girouard, notaire: "Elle a réuni un groupe de femmes pour prendre ensemble des résolutions à l'effet de concourir, autant que la faiblesse de leur sexe le leur permette, à faire réussir la cause patriotique". Le Comité central et permanent approuve pleinement ces dignes femmes et leur permet de se former en "Association des dames patriotiques du comté des Deux-Montagnes".

1. Comité fondé en 1834. C'était, pour les "patriotes", l'organisme central d'information et de direction. 
C'est dans la maison de Madame Girouard qu'ont lieu les assemblées politiques du comté des Deux-Montagnes; c'est sur sa maison qu'on arbore un drapeau, portant ces mots encadrés de trois têtes de mort: "Conseil Législatif". C'est encore là que les Patriotes se réunissent pour convenir des meilleurs moyens d'organiser militairement le comté des Deux-Montagnes.

"A ces assemblées de Comités se fesaient aussi des discours, pour encourager \& exhorter le peuple à s'en tenir strictement au "système de non-consommation". Cette partie de notre résistance constitutionnelle \& passive aux tyrans réussit d'une manière étonnante. Hommes, femmes, enfants, tous travaillaient avec ardeur à diminuer le revenu. Les Représentans, furent les premiers à donner l'exemple, \& à paraître en public vêtus d'étoffes Canadiennes. Les jeunes gens ne restèrent pas en arrière. La vanité, l'amour de la toilette, qui leur est souvent naturelle, ne les empêcha pas de porter la grosse toile \& l'étoffe grise. Salus Patriae Suprema lex est! Le beau sexe ne les repoussa pas sous le costume national. Au contraire, à St-Antoine, St-Denis, St-Charles, comté des Deux-Montagnes, etc. elles eurent des assemblées, dans lesquelles elles promirent de repousser les ennemis de leur pays, \& de donner la préférence $\&$ une place dans leurs cœurs, à ceux qui n'auraient pas honte de porter les tissus qu'elles fileraient de leurs propres mains. A Montréal, Mad. Lafontaine \& Mad. Peltier eurent l'honneur d'être les deux premières dames, qui parurent publiquement vêtus d'étoffes canadiennes. Cette mesure doubla, tripla la valeur des produits domestiques, \& par conséquent enrichit le cultivateur, et fit du bien au pays, en même temps qu'elle fesait du mal à ses oppresseurs. Chaque jour voyait paraître dans le marché de nouveaux patrons, et d'étoffes plus fines"'.

Dans la région du Richelieu, à l'automne de 1837, lors des assemblées qui s'y tiennent, on remarque, parmi les drapeaux, un fanion qui porte cette inscription: "Honneur aux Dames Patriotes".

Pour comprendre l'état d'esprit de ces femmes, il est intéressant de lire une lettre de Cordélia Lovell à sa sœur madame Allard, datée

2. Extrait de: "Journal d'un Fils de la Liberté réfugié aux Etats-Unis par suite de l'insurrection canadienne en 1837", par Louis-Joseph-Amédée Papineau, fils ấé du grand tribun patriote Papineau. L'extrait suivant a paru dans La Presse, (Montréal, samedi, 26 janvier 1924). 
du 11 novembre; elle fait part à sa correspondante de ses inquiétudes sur les mouvements politiques de l'heure:

...Nous avons reçu une lettre du Dr. Dorion toute remplie par les événements politiques. Le docteur dit que tout est calme à Saint-Ours, mais tel n'est pas le cas à Chambly, particulièrement, depuis l'arrivée des troupes, ce qui a eu pour effet de créer un plus vif mécontentement. Il paraît qu'il y a eu des troubles sérieux à Montréal, et le docteur eraint que cela cause encore plus de confusion à Saint-Charles avant peu. J'ai appris que vous aviez été témoins de ce qui est arrivé à $M$. Debartzch à son retour de Québec. Il est très malheureux que le docteur s'engage si profondément dans la politique, il ne me paraît pas assez prudent. J'aimerais bien savoir ce que M. Allard pense là-dessus et s'il appréhende des troubles sérieux pour cet hiver. A Québec tout paraît aller paisiblement mais il pourrait bien se faire qu'on prépare quelque organisation... A me lire vous devez croire que je suis devenue politicienne. Non, assurément, mais je dois vous dire qu'il ne m'est pas possible de me désintéresser des événements qui se produisent... Les affaires de la politique occupent tout le monde, c'est le sujet de toutes les conversations... ${ }^{3}$

Une femme, qui ne craint pas les armes à feu, à cette époque, c'est madame Timothée Kimber, épouse du docteur Kimber ${ }^{4}$. Établi à Chambly à l'époque de la rébellion, le docteur s'engage à fond dans le mouvement. Il a épousé, à Chambly, le 12 novembre 1822, Émilie Boileau, fille de René Boileau et de Marie-Antoinette de Gannes de Falaise. Si l'on s'en rapporte aux mémoires de Bouchette, madame Kimber aurait été une personne décidée et prête à tout. Passant par Chambly, en route vers les États-Unis, Bouchette insiste pour arrêter un instant chez le Dr Kimber, quoique celui-ci fât absent. Voici comment il raconte la réception qu'on lui fit:

...Mon ami Drolet me servit de passeport et nous nous rendîmes à destination sous la garde d'une autre sentinelle. Après beaucoup d'hésitation, on nous permit de franchir le seuil de la porte, et nous fûmes admis dans une grande salle où

3. Abbé A. Couillard-Després, Histoire de la famille et de la seigneurie de SaintOurs (2 vol., Montréal, 1917), 2: 286-287.

4. Aegidius Fauteux, Patriotes de 1897-1898 (Montréal, 1950), 277. 
se trouvait beaucoup de monde. A peine y étions-nous entrés, que nous vîmes les personnes qui occupaient le fond de la salle se diviser respectueusement pour laisser passer une dame qui s'avançait vers nous avec calme et dignitê. Elle tenait dans sa main droite un pistolet dont le canon reposait sur son bras gauche. M. Drolet me présenta à madame Kimber...

Le docteur Chénier a porté le drapeau des Deux-Montagnes, le 23 octobre 1837, à la grande assemblée de Saint-Charles. Le comté des Deux-Montagnes connut un autre drapeau, à la bataille de Saint-Eustache. Les patriotes y arborent un drapeau dit canadien: drapeau blanc, traversé d'une branche d'érable, portant une couronne de cônes et feuilles de pin de couleur bleue, entourant un poisson brun avec les lettres $\mathrm{C}$ pour Canada et J.-Bte indiquant la race canadienne-française. Ce drapeau fut dessiné et tissé par les dames Masson et Dumouchel pour les combattants de Saint-Eustache au mois de décembre 1837. Ces deux dames ont donc conçu et exécuté le premier drapeau canadien-français. On peut le voir aujourd'hui au Château de Ramezay.

Pour préparer la journée du 14 décembre 1837, les patriotes ont fabriqué des épées et des poignards. Une centaine d'entre eux, tout au plus, possèdent de mauvais fusils ${ }^{6}$. Les demoiselles Labrie et Berthelot entreprennent de leur fondre des balles.

Madame Julien Perrault, née Euphrosine Lamontagne, était la mère de Charles-Ovide Perrault, avocat et député de Vaudreuil. Atteint, le 23 novembre 1837, à la bataille de St-Denis, d'une balle à l'abdomen et d'une autre au talon, Perrault mourut le lendemain. Son frère, Louis Perrault, était l'imprimeur du Vindicator. Voici ce que nous apprend une lettre de L.-J. Papineau à Louis Perrault:

Albany 7 janvier 1839.

...Pendant que nous étions à Washington le Consul Buchanan y est venu loger dans la maison ou nous étions. Étaitil franc et sincère. Il a ouvertement censuré la conduite de Durham à notre égard et disait beaucoup de bien des Canadiens

5. Mémoires de Robert-S.-M. Bouchette 1805-1840. Recueillis par son fils Errol Bouchette et annotés par A.-D. Decelles. (Montréal, 1903), 44.

6. Dr J.-B. Richard, Les événements de 1837 a Saint-Denis-sur-Richelieu (StHyacinthe, 1938) Documents maskoutains, no 2: 34 . 
et paraissait s'indigner des excès et de la soif de sang de la faction. Il me disait combien il avait admiré et combien il respectait l'énergie qu'avait déployé votre excellente et respectable Mère. Il me disait d'elle, que lors de leur entrevue sur le Steamboat, et lorsqu'elle se rendait pour vous aller voir à Burlington, il lui avait dit madame ne blamez vous pas ce qui s'est passé en Canada. Ces événements vous ont fait perdre un fils que vous pleurez si justement, ils en condamnent un autre à l'exil, rien rien de cela ne serait arrivé si les gens étaient demeurés tranquilles. Mais Qu'elle l'avait repris avec vivacitê. Mr. je blâme le gouvernement qui a forcé des citoyens vertueux à la nécessité de se défendre, je déplore profondément les pertes que j'ai souffertes, mais si c'était à recommencer et que mes enfants voulussent agir comme ils l'ont fait je n'essayerais pas à les détourner parce qu'ils n'agissent nullement par ambition, mais par amour du pays et par haine contre les injustices qu'il endure. Il m'ajoutait j'ai écrit les détails de cette entrevue en Angleterre pour leur faire sentir la nécessité d'user de modération à l'égard d'un Pays dont la nationalité est si prononcée. Il m'enjoignait le secret je vous l'enjoins aussi et ne vous en fait part et à votre mère que comme un juste tribut de respect qui lui est dû par tous et qu'il lui est rendu par un adversaire politique ce qui me donnait un grand plaisir et doit vous être de quelque consolation?.

\section{II - Elles ont souffert}

“...dans la soirée, [ du 14 déc. 1837 ] raconte Émélie Berthelot, d'autres volontaires revinrent dans la Grande-Côte, chez un nommé Maisonneuve, et se faisant donner de l'eau, emplirent les canons de leurs fusils et burent à même. Étant assez en train ils entrèrent de nouveau chez le père Rochon, cassèrent les vitres avec leurs baïonnettes, fouillèrent les armoires et se chargèrent de linge. Ils reprenaient la route du village, dira-t-elle, lorsque mon père tenta d'arrêter leur brutalité. Mais ils le prirent sous les bras et l'emmenèrent au village. Ma bonne mère, toute en larmes, me conjura de l'aller chercher. Je sortis et rejoignis les volontaires. Je les suppliai de relâcher mon père. Au cours de mes larmes, je leur dis: "Si vous aviez

7. Extrait d'une lettre de L.-J. Papineau à Louis Perrault, imprimeur du Vindicator, Arch. du Can. Papiers Perrault, 1838-1839, 6. 
votre père pris par les soldats et emmené par eux, ne seriez-vous pas émus ?" Ils me regardèrent étonnés et poussèrent mon père de mon côté en disant: "C'est bien, va-t-en!" Et des scènes semblables se renouvelèrent par dizaines, par vingtaines, dans les rangs du Lac, du Grand-Brâlé et du Domaine ${ }^{8}$. Chaque fois, la Canadienne est là pour défendre les siens contre l'ennemi, elle ne perd jamais courage.

Dans une maison nouvellement bâtie, au fond de la grande anse des Éboulis, plusieurs femmes se sont réfugiées avec quelques couvertures soustraites aux envahisseurs. Un grand nombre de jeunes filles ont trouvé refuge dans la maison de ferme du Séminaire de St-Sulpice à la Pointe-des-Anglais, "pour se soustraire aux poursuites et aux brutalités des loyaux et des soldats".

Après avoir pillé tout ce qui se trouve dans les maisons et les bâtiments des fermes et s'être emparés des animaux, les bureaucrates font déshabiller les hommes, les femmes et les enfants, qu'ils laissent presque nus à la porte de leurs maisons en flammes ${ }^{10}$. Les dames Dumouchel et Lemaire, Girouard et Masson ne sont pas exemptes des assauts de ces messieurs. Les bureaucrates savent qu'elles sont les épouses des plus ardents patriotes. Ils en profitent pour leur laisser à peine de quoi couvrir leur nudité. Personne ne sait comment ces infortunées purent survivre à tant de misères. On avait défendu aux habitants de donner l'hospitalité à ces pauvres femmes; elles seraient mortes de froid, sans le courage de quelques citoyens, qui leur offrent le gîte au risque de la vindicte loyaliste. Cependant deux jeunes filles ne peuvent surmonter l'épreuve. Olive Lemaire meurt quelques jours plus tard; Cléophée Masson tombe dangereusement malade.

Parmi ces femmes qui fuient, il faut en distinguer deux, madame Damien Masson, épouse du docteur Masson, un des chefs patriotes de St-Benoît, et la sœur du Dr Chénier. Madame Masson a pris le parti de fuir avec ses quatre plus jeunes enfants. Avant de partir, et, après avoir mis en sûreté quelques-uns de ses meubles, ses provisions de ménage, elle prend le chemin de la Côte Saint-Vincent;

8. Abbé Emile Dubois, Le Feu de la Rivière-du-Chêne (Montrésl, 1937), 152.

9. Ibid., 197.

10. L.-O. David, Les Patriotes de 1897-1888 (Montréal, 1913), 61. 
elle emporte ses livres de compte et un chien qu'Adéline porte dans ses bras. Un cultivateur, Antoine Lalonde, qui voit passer les fugitives, grelottantes de froid et harassées de fatigue, les loge chez lui, au risque de représailles. C'est dans cette demeure que madame Masson fait une rencontre tragique. Un jour, elle voit arriver une femme harassée de fatigue. Elle vient de Rigaud; elle a traversé le lac des Deux-Montagnes, et parcouru le chemin pour s'enquérir du sort de son malheureux frère, le docteur Chénier. Madame Masson a tôt fait de reconnaître son amie de Rigaud. Toute en larmes, elle lui annonce la mort de son frère. A son tour, la sœur de Chénier raconte à Mme Masson l'arrestation, au Côteau-du-Lac, de Luc et de Damien, ses deux fils." 11

A St-Benoît, comme à St-Eustache, les familles des patriotes furent jetées sur la grand'route.Les côtes Saint-Louis, Saint-Joachim, Saint-Vincent et Saint-Jean des Deux-Montagnes sont littéralement saccagées. Dans cette dernière les loyalistes poussent la cruauté jusqu'à arracher brutalement à une dame Benjamin Maynard, le lit où elle repose avec un enfant de deux jours dans les bras. La pauvre femme subit un tel choc nerveux qu'elle en meurt le lendemain. ${ }^{12}$

Dans plusieurs autres villages, les femmes vont connaître un sort semblable. Quelque temps après l'affaire de Napierville, les volontaires ordonnent à Mme Merizzi de sortir de sa maison, qu'ils vont incendier. Elle s'y refuse. Les brigands mettent le feu. La dame persiste à demeurer; elle se laissera brûler, dans sa maison, proteste-telle, plutôt que d'en sortir. Étonnés de la fermeté héroïque de cette femme, les volontaires éteignent le feu; la maison est sauvée ${ }^{13}$. Dans bien des endroits l'on met le feu indistinctement à toutes les maisons.

Aux environs de Napierville, dans les bois de Lacolle, une femme fut trouvée assise au pied d'un arbre, un enfant dans ses bras et deux à ses côtés, tous quatre morts de faim et de froid. Une lettre du docteur Davignon confirme ce trait. Cette femme était

11. Ibid., 172.

12. Gérard Filteau, Histoire des Patriotes (3 vol., Montréal, 1939), 3: 104.

13. Extrait de: "Journal d'un Fils de la Liberté réfugié aux Etats-Unis par suite de l'Insurrection canadienne en 1837 par Louis-Joseph Papineau, fils ấné du grand tribun patriote Papineau" (5 vol., texte dactylographié à la Municipale de Montréal) 3: 398 . 
accouchée là au pied d'un arbre, de l'enfant qu'elle tenait sur elle. Dieu certes est venu abréger les souffrances de cette martyre de la brutalité des hommes. ${ }^{14}$

Je ne puis terminer cette longue liste des souffrances des Canadiennes, innocentes victimes des troubles politiques de l'époque, sans rappeler ici la conduite exemplaire de madame Julien Gagnon, née Regnier, de St-Valentin. Julien Gagnon, ou Gagnon l'habitant comme on l'appelait, était un cultivateur à l'aise de la Pointe-à-la-Mule, paroisse de Saint-Valentin.

Un soir que madame Gagnon était seule avec ses enfants, des hommes armés entrent soudain dans sa maison, l'insultent, la menacent, lui annoncent qu'ils viennent au nom de la reine confisquer tous les biens de son mari, s'emparent en effet de tout, clouent les portes de toutes les chambres de la maison, des granges, bâtiments et dépendances, et donnent trois heures à madame Gagnon pour sortir avec sa famille. La pauvre femme essaya en vain de toucher ces barbares en leur montrant ses huit enfants pressés autour d'elle, et sa vieille mère âgée de soixante-quinze ans; elle leur demande même en vain la permission d'emporter des vêtements et des provisions. Elle fut obligée de partir, dénuée de tout. Et l'on vit cette pauvre femme sur le chemin, par une nuit noire et froide, aller de porte en porte, un enfant dans les bras, suivie d'une vieille femme de soixante-quinze ans, sa mère, et de sept enfants, tremblants de peur, grelottants de froid. Les bureaucrates avaient tellement effrayé le voisinage, qu'à plusieurs endroits on ne veut pas recevoir la femme et les enfants de Gagnon. Les fugitifs furent donc obligés de faire une demilieue avant de trouver un refuge. Quelques jours après, Mme Gagnon et sa famille prenaient la route des États-Unis pour faire son triste voyage; elle s'en allait, le cœur serré, mais confiante et certaine qu'on la laisserait passer tranquille. Vain espoir! Elle était à peine partie, qu'une troupe de bureaucrates l'attaquait, pillait les voitures, s'emparait de tout, vêtements et provisions, et la laissait à peine vêtue sur le grand chemin ${ }^{15}$.

Cependant la pauvre femme réussit enfin à franchir la frontière et à rejoindre son mari. Mme Gagnon passe une partie de l'hiver

14. Ibid., 3: 399.

15. L.-0. David, Les Patriotes de 1837-1838 (Montréal, 1913), 106. 
avec son mari, à Corbeau, à quelques milles de la frontière. Au mois de mars, cette femme courageuse voit sa famille sans ressources. Elle entreprend alors de revenir au Canada pour reprendre possession du bien des siens et essayer d'ensemencer la terre. Elle réussit, avec l'aide de ses enfants et de quelques voisins, à semer quelques minots de grains. Gagnon brave le danger qui le menace; il vient voir sa famille, la nuit, a travers bois ${ }^{16}$. Un soir, un courrier apprend à Gagnon que Robert Nelson lui donne rendez-vous à Napierville.

Il part avec l'intention de revenir pendant la nuit. Il a été vu, un traître le dénonce. Vers onze heures, un grand bruit se fait autour de la maison; ce sont des dragons qui arrivent dans l'espoir de le surprendre. Ils enfoncent les portes, crient, jurent, fouillent partout et ne trouvant pas celui qu'ils cherchaient, veulent savoir où il est. Ils s'adressent à l'aîné des fils de M. Gagnon, et veulent le faire parler; comme il refuse, ils se précipitent sur lui, le garrottent et le soumettent à toutes sortes de mauvais traitements. Ils percent de plusieurs coups de baïonnette son frère Jules, et brisent, d'un coup de crosse de fusil, l'épaule de la mère de madame Gagnon, une pauvre vieille de soixante-quinze ans ${ }^{17}$.

Tout ceci se passe sous les yeux de madame Gagnon. Dans son affolement, elle pense à son mari qui doit revenir; elle envoie l'aînée de ses filles, âgée de douze ans, guetter son père. La pauvre enfant passe le reste de la nuit blottie près de la clôture sur le bord du chemin. Enfin, le jour arrivé, les dragons évacuent la maison, après avoir brisé une partie des meubles, mais non sans promettre de revenir bientôt. Madame Gagnon comprend que sa vie et celle de ses enfants sont en danger; le lendemain elle reprend le chemin des États-Unis.

Une compatriote de madame Julien Gagnon, Madame Théophile Barbeau, a marché de St-Hyacinthe jusqu'à Albany, pieds nus. Louis-Joseph Papineau fils nous dit, dans son "Journal", que: "le samedi ler juin 1838, six familles canadiennes arrivent vers trois heures à Platsburg, elles sont de St-Hyacinthe et de Ste-Marie. Parmi eux est une veuve avec 4 enfants, dont l'aînée, une fille, a neuf ans

16. Ibid., 108.

17. Ibid., 108-109. 
et dont le père, Théophile Barbeau, tomba sous le plomb meurtrier des tyrans à la bataille de St-Charles. La pauvre femme était nupieds. Elle vient de St-Hyacinthe et se rend à Albany"18.

Extrait d'un article du journal "La Quotidienne:" “On nous dit que le jour de Noël, vers quatre heures du matin, trois vieilles femmes ont été arrêtées à la porte de la ville, au bout du faubourg Québec. Elles venaient pour la messe du point du jour, armées de leurs chapelets et de leurs livres de prières. Elles furent détenues assez longtemps pour perdre la messe, à leur grand regret." Le rédacteur ajoute malicieusement; "elles persistent à dire qu'elles n'avaient aucune intention de prendre la ville."

Citons le notaire Girouard, de Saint-Benoît qui écrivait à son ami A.-N. Morin, le 9 mars 1838:

...Si tu vois Madame Mongrain, n'oublie pas de lui témoigner combien je me rappèle avec sensibilité l'intérêt qu'elle m'a porté lorsque j'étais gardé par les braves femmes patriotes dans le grenier de la maison de Payen... Quelle scène que celle-là! je l'ai toujours à la mémoire. Si jamais je retourne à St-Benoît, et que j'en aie les moyens je vais rassembler avec nous toutes ces généreuses femmes que les promesses, l'argent, la crainte, n'ont pu engager à trahir un de leurs compatriotes. Je voudrais avoir une occasion de les remercier, de leur témoigner ma reconnaissance, et l'admiration que j'ai pour leur patriotisme. Du moins j'écrirai ces actes de vertu. Je ne me rappèle pas le nom de toutes les femmes qui se trouvaient dans cette maison, qui m'a servi de dernière retraite, mais ne manque pas de remercier toutes celles que tu rencontreras. La bonne Esther pourra peut-être te les nommer. Sans ces généreuses patriotes, j'eusse inévitablement tombé entre les mains des affreux cannibales qui ont porté la flamme et la désolation chez nous; ils m'auraient égorgé, et tu n'aurais pas aujourd'hui l'espoir d'embrasser encore ton affectionne ${ }^{19}$.

Et encore, le 6 novembre 1841 :

$$
\text { J.-J. Girouard }
$$

...Dans ces temps malheureux les femmes seules se montrèrent au-dessus des circonstances et soutinrent un courage que le sombre despotisme voulait entièrement abattre ${ }^{20}$.

18. Louis-Joseph-Amédée Papineau, "Journal d'un Fils de la Liberté", 3: 353.

19. Extrait d'une lettre de J.-J. Girouard à sa femme (inédite). Montréal, Nouvelle Prison, 9 mars 1838.

20. Ibid., 6 novembre 1841. 


\section{III - Elles ont consolé}

A la bataille de Saint-Denis, du côté des Anglais, il y a une trentaine de blessés, dont huit faits prisonniers. Ces malheureux sont bien convaincus, après ce qui a été dit, qu'ils seront massacrés sans pitié par leurs vainqueurs; aussi ils sont agréablement surpris de voir les femmes de Saint-Denis rivaliser à qui leur prodiguera les meilleurs soins. Les femmes de Saint-Denis ont une attitude chrétienne. Elle ne font aucune distinction de race ou de religion parmi les blessés, elles les secourent également. Les blessés anglais sont transportés chez les demoiselles Darnicourt; aidées de quelques amies, celles-ci les traitent avec délicatesse et un dévouement qui les touchent profondément. Les demoiselles Darnicourt sont les filles du docteur Darnicourt.

Lorsque, huit jours après, les vaincus du 23 novembre reviennent à Saint-Denis, pour venger leur défaite par le pillage et l'incendie, les demoiselles Darnicourt s'adressent au colonel Gore pour le prier d'épargner le village. Mais les soldats et les volontaires s'acharnent à mettre le feu partout. Cependant ils reconnaissent ce que les demoiselles Darnicourt ont fait pour leurs blessés; ils épargnent leur maison, celle de leur voisine, mademoiselle Chalifou, et une grange qui renferme toute la récolte de madame St-Germain.

Le soulèvement écrasé, c'est l'heure de la répression. Les autorités procèdent aux arrestations. C'est dire que les femmes tombent dans un état d'angoisse indescriptible. Mais encore là les Canadiennes cherchent à s'entr'aider, à se consoler. Pour mieux comprendre la manière dont la plupart des femmes réagissent, lisons ce que madame Pierre Dorion écrit à sa sœur, madame Catherine Lovell Dorion, le 19 décembre 1837:

Ma chère Kitty,

J'ai reçu avec plaisir la lettre de cette bonne Eliza, j'avais tant d'hâte de recevoir des nouvelles, je savais que le docteur était détenu à Montréal, je pensais bien dans quel état tu pouvais être ma bonne sœur. Je sais comme cela doit t'être sensible mais comme tu vois c'est le sort de tous ces hommes qui se sont Mêlé de Politique. Mais je t'assure que je n'étais pas bien inquiète pour le Docteur parce que je sais qu'il a trop d'esprit pour s'être laissé entraîner comme tant d'autres. Mais seulement qu'il s'est rendu suspect ayant figuré dans les assem- 
blées ${ }^{22}$. Monsieur Allard a eu la bonté de nous écrire de Montréal et nous fait espérer que ton mari te sera bientôt rendu, tâche, ma chère Kitty de supporter ceci avec courage; pense à tes enfants, à ta situation et surtout à tous ces pauvres femmes dont les maris sont si éperduement lancés dans la politique qu'il [ leur ] faut pour se sauver, s'exiler de leur patrie! Je n'ai pas besoin de te dire de supporter ceci en chrétienne; je connais ta soumission aux décrets de la Providence. Je prie Dieu tous les jours qu'il t'accorde la santé et le courage nécessaires pour supporter ta peine, et pour ton mari, qu'il soit au plus tôt rendu à sa famille et pardessus tout qu'il ne se mêle plus de politique. Combien j'aurais désiré d'être près de toi pour te consoler, j'étais si inquiète et si misérable que je ne savais que faire, combien j'étais donc heureuse d'apprendre qu'Eliza était avec toi elle qui est si capable de t'encourager. Quel changement depuis deux mois, espérons des jours plus heureux. Tout ici est bien tranquille. Mais toutes les précautions possibles sont prises pour plus grande sûreté on ne rencontre qu'officiers et soldats volontaires partout. Toutes les portes de la ville se ferment tous les soirs de bonne heure. Eliza est-elle encore avec toi ? je l'espère; embrasse-la bien tendrement pour moi et dis-lui combien je suis reconnaissante pour sa lettre, des baisers pour tes chers enfants, encore une fois du courage ma bonne Kitty, j'espère que tu voudras bien nous laisser savoir aussitôt que le docteur sera à St-Ours, que dis-je? il y est peut-être déjà? dans cet heureux espoir, je termine, ma chère sœur, puisses-tu oublier ces jours d'amertume et jouir du bonheur que tu mérites; c'est le vœu de mon cœur.

Cordélia ${ }^{21}$.

La lutte terminée, sur les champs de bataille, une autre va commencer, devant la Cour Martiale.

Parmi les premières femmes à porter secours aux prisonniers, il Ifaut citer madame Papin, de Lachine et sa fille. Ces dames se rendent à la prison à la Pointe-à-Callières, une sorte de hangar érigé en prison; elle y apportent des provisions préparées de leurs mains charitables, elles encouragent et sympathisent avec les prisonniers ${ }^{23}$.

21. Abbé A. Couillard-Després, Histoire de la famille et de la seigneurie de SaintOurs (2 vol., Montréal 1915-1917), 2: 287-288.

22. On constate ici que les femmes ne sont pas toujours bien renseignées, car le docteur Dorion prit une part très active à l'insurrection de 1837.

23. F.-X. Prieur, Notes d'un Condamné Politique de 1888 (Montrésl, 1884), 43. 
La plus remarquable pour sa bonté, et qui recevra, un jour, le surnom d'Ange des prisonniers, est sans contredit, Mère Gamelin. Toujours prête à soulager infortunes et douleurs nouvelles, Mme Gamelin ne limite pas son dévouement et son zèle au service des pauvres de son asile et de son quartier. L'insurrection de 1837 lui fournit l'occasion d'en donner la preuve. La prison de Montreal regorge de détenus politiques, tant de la ville que de la campagne; un grand nombre appartiennent à de bonnes familles; plusieurs ont femme et enfants, avec lesquels il leur est rigoureusement défendu de communiquer. Madame Gamelin s'émeut de leur souffrance et s'occupe à y porter secours.

Vers 1900, la visite des prisonniers se fait encore par les filles de madame Gamelin. Les sœurs accompagnent même les condamnés au pied de l'échafaud. Aujourd'hui, en 1925, les sœurs de la Providence accompagnent les femmes condamnées à mourir sur l'échafaud. Il est dans leur ordre d'accompagner les condamnés, elles ne le font plus, parce qu'elles ne sont pas demandées. Mais il est certain que si un condamné insistait, un jour, pour être accompagné par des religieuses, les sœurs de la Providence, au nombre de deux, assisteraient à l'exécution ${ }^{24}$.

Madame Gamelin sollicite des autorités et obtient sans peine, un permis général pour pénétrer auprès des détenus, et leur porter secours chaque fois qu'elle le juge à propos. Elle profite largement de l'autorisation; chaque jour, on peut la voir, un panier au bras, rempli de provisions, accompagnée d'une dame, qui est le plus souvent madame Gauvin, mère du docteur Gauvin, nóe Marguerite Barsalou, franchir le seuil, salué au passage par les factionnaires anglais qui lui présentent les armes. La faveur accordée à madame Gamelin est bientôt connue; la dame charitable reçoit donc sans cesse visites ou lettres des parents et amis des captifs qui lui confient leurs messages et leurs dons. Mme Gamelin s'en charge avec bonheur.

Voici une anecdote de ce ministère de charité. L'héroïne est une enfant de dix ans, future religieuse de la Providence, sœur Jean-Bap143.

24. Par une religieuse de l'Institut, Vie de Mère Gamelin (Montréal, 1925), 
tiste. Son père Jacques Longtin, cultivateur de St-Constant, est au nombre des détenus. Le 8 novembre 1838, elle vient à Montréal avec sa mère, pour tenter de pénétrer auprès de son père. La permission leur est refusée; leur douleur est grande; la loi martiale à été proclamée la veille même; des rumeurs sinistres circulent sur le sort réservé aux détenus. Dans son affliction, la pauvre femme se rend chez Mme Gamelin pour lui demander conseil et assistance. "Celle-ci, raconte sœur Jean-Baptiste, qui ne peut amener ma mère à la prison, à cause du refus qu'elle vient d'essuyer, a la délicatesse de me prendre avec elle pour sa visite quotidienne. Je pars donc avec madame Gamelin, je lui aide à porter ses provisions dont une part est destinée à mon père. J'ai le cœur bien gros, et des larmes coulent le long de mes joues, en songeant que je vais voir mon père, prisonnier, lui, que nous aimons tant! Nous traversons la cour de la prison entre deux rangées de soldats armés. Le guichetier ouvre une grande porte en fer et la referme sur nous. Je tremble de tous mes membres, mais madame Gamelin me rassure avec une bonté toute maternelle."

"Quelques instants après, nous étions dans la salle des détenus. En apercevant Mme Gamelin, les prisonniers viennent la saluer. Elle leur rend leur salut en disant: "Je viens voir comment se portent mes enfants aujourd'hui!" Pendant qu'elle leur distribue les messages de leurs familles et ses provisions, parmi lesquelles il y a du tabac et des friandises, je peux voir mon bon père. Je ne sais ce que je lui dis, mes sanglots m'étouffent; cette entrevue est restée pour toujours gravée dans ma mémoire. (Jacques Longtin fut condamné à mort, puis exilé en Australie.) "Durant cette visite, madame Gamelin fait aux prisonniers une courte lecture de piété, comme elle le fait toujours; elle récite le chapelet avec eux et, sur le point de partir, leur dit en souriant: "Si vous voulez bien, avant que je me retire, nous allons faire ensemble notre prière du soir." Et tous ces braves gens, s'agenouillent sur les dalles et répondent tout bas à la voix de madame Gamelin. Le souvenir de son dévouement n'a pas été perdu pour l'histoire. Plusieurs ouvrages publiés en font mention.

Ne quittons pas madame Gamelin sans dire un mot de celle qui lui a apporté une aide très précieuse, madame Gauvin ${ }^{25}$. Ses filles, les

25. L.-O. David, Les Patriotes de 1837-1898 (Montréal, 1925), 143. 
demoiselles Gauvin, ont beaucoup aidé leur mère dans son œuvre charitable auprès des prisonniers.

Le 21 décembre 1838, Joseph Duquet, jeune homme dans la vingtaine, monte sur l'échafaud avec Cardinal, député de Laprairie. Démarches et requêtes nombreuses affluent chez Colborne; mais le Vieux Brulôt reste inexorable.

Les derniers jours de Duquet sont particulièrement tristes. Il est jeune, il ne peut se résigner à mourir. Sa mère, née Marie-Louise Dandurand, et sa sœur, le voient souvent, essayent de le consoler, de lui faire accepter la mort. Jusqu'au dernier moment, Mme Duquet supplie le gouverneur d'épargner son fils, elle descend même à Québec pour lui remettre une lettre en mains propres, mais sans succès.

On ne peut, non plus, passer sous silence le nom de Madame Cardinal. Joseph-Narcisse Cardinal avait épousé, à Montréal, en 1831, Mlle Eugénie Saint-Germain, Le couple fut très heureux; de ce mariage sont nés cinq enfants. Cardinal s'est mêlé avec ardeur à l'insurrection de 1838; il exerce l'autorité suprême dans le village de Châteauguay. Le 3 novembre il est à la tête des patriotes qui se rendent au village de Caughnawaga pour s'emparer des armes des Indiens. Nous connaissons le résultat de cette triste expédition, l'arrestation de Cardinal et de ses compagnons, leur procès, leur condamnation. Le 8 décembre 1838, Cardinal est condamné à mort. De grands efforts sont faits par des personnes influentes pour obtenir la grâce de Cardinal ou la commutation de la terrible sentence. Colborne résiste à toutes les instances. Dans l'espoir qu'une femme sera plus sensible à sa douleur, Mme Cardinal écrit à Lady Colborne la lettre suivante ${ }^{26}$ :

My lady,

Vous êtes femme et vous êtes mère! Une femme, une mère poussée par le désespoir, oubliant les règles de l'étiquette, qui la séparent de vous tombe à vos pieds, tremblante d'effroi et le cour brisé, pour vous demander la vie de son époux bienaimé et du père de ses cinq enfánts! L'arrêt de mort est déjà signé! L'heure fatale approche!

Demain, hélas! demain!!! Dieu! ô Dieu! Je n'ai pas la foree d'envisager un sort aussi horrible. La seule pensée que j'en ai remplit mon âme de désespoir: que sera la réalité?

26. Ibid., 203-204. 
Oh! je ne pourrai jamais supporter une pareille calamité! Le coup qui tranchera le fil de ses jours, nous frappera tous deux. Je serais plus forte si une autre existence ne dépendait pas de la mienne! Mais mon malheureux enfant ne verra jamais la lumière du jour! Il périra avec sa mère sous l'échafaud où son père, qui méritait un meilleur sort, aura péri. O Dieu! est-ce ainsi que vous punissez? Non, non, pardonnez-moi ce blasphème. Les hommes seuls ont recours à de telles vengeances. Les hommes seuls font perir l'innocent avec le coupable... coupable... Que dis-je? Et mon mari, de quoi s'est-il rendu coupable? Le plus qu'on puisse dire, c'est qu'un peu d'excitation, de faiblesse peut-être, l'a perdu. Son ennemi juré celui qui avait résolu sa mort... est le même homme qui n'a pu le convaincre d'un seul acte de violence! Faut-il que son sang soit répandu, lui qui loin de répandre le sang de ses semblables n'a jamais causé le moindre tort dans tout le cours de sa vie? Car c'est une atroce calomnie de dire qu'il a causé la ruine des autres. D'un caractère très timide, fréquentant peu la société - ne jouissant de la vie qu'au milieu de sa famille qui l'adorait - il n'a pris aucune part à l'agitation qui a précédé les dernières scènes de malheur. C'est donc dans sa maison qu'il a été surpris par un mouvement soudain et non prévu. Il n'a pas fait de victimes, au contraire, il est lui-même victime. Voilà tout son crime, et ce crime, (si c'en est un) ne l'a-t-il pas déjà expié? N'a-t-il pas déjà trop souffert? Et durant tout le temps de sa détention dans son cachot solitaire, négligée de tous, nous, votre humble requérante et ses enfants, n'avons-nous pas souffert suffisamment pour lui? Autrefois, heureux avec lui, bien que de condition humble, n'avons-nous pas été bannis de notre demeure par la torche et la brutalité de l'incendiaire? N'avons-nous pas été dépouillés de tout, même de nos vêtements? N'avons-nous pas été obligés de vivre du pain provenant de la bonté du Très-Haut, et qui nous était donné par les personnes charitables, qui pour l'amour de Dieu, prennent plaisir à le distribuer à ceux qui sont dans le besoin. Et vous, Mylady, quel trésor le ciel n'a-t-il pas mis entre vos mains? Ne vous a-t-il pas donné une influence immense sur l'esprit et le cour qui aujourd'hui gouverne nos destinées? Faites comme les personnes charitables dont je viens de parler, servez-vous de ce trésor pour votre avantage dans l'éternité, pour celui de l'époux que vous chérissez et des enfants qui font votre gloire et votre bonheur. Oh! l'humanité n'est certainement pas bannie de cette terre de vengeance, elle doit s'être refugiée dans le cour des femmes, sans doute, dans le cœur des mères, comme le vôtre. L'humanité parlera 
par vos lèvres, - elle sera persuasive, éloquente, irrésistible, elle arrêtera le glaive de la mort, prêt à immoler tant de victimes, elle apportera la joie dans le cour de tant d'infortunés qui redoutent le lever du soleil de demain, elle sera entendue même dans le ciel et sera inscrite à votre crédit dans le livre de vie.

$$
\begin{gathered}
\text { J'ai l'honneur d'être, } \\
\text { Mylady, } \\
\text { votre très-humble et affligée servante, } \\
\text { Eugénie Saint-Germain, } \\
\text { épouse de Joseph-Narcisse Cardinal. }
\end{gathered}
$$

Madame Cardinal attend la naissance d'un enfant. Ne recevant pas de réponse à sa lettre, elle va quand même voir lady Colborne à Québec. De vive voix elle implore son intercession en faveur de son mari. Peine inutile! Lady Colborne lui présente huit piastres! La Canadienne indignée se lève et lui fait cette fière réponse: "C'est la vie de mon mari que je demande, ce n'est point votre argent" ${ }^{27}$.

Cardinal a perdu l'espoir de voir, avant de mourir, sa femme et ses enfants. La. veille de son exécution, tard dans la soirée, on se ravise et on lui accorde la permission demandée. Cardinal s'efforce de se maîtriser, de paraître résigné. Quand l'heure de la séparation sonne à l'horloge de la prison, le condamné et sa femme se font leurs adieux, plus morts que vivants ${ }^{28}$.

La dernière figure émouvante, parmi les femmes des prisonniers politiques, dont il faut rappeler la conduite courageuse, est bien madame Henriette Chevalier de Lorimier, née Henriette Cadieux, fille aînée de Jean-Marie Cadieux, notaire à Montréal.

Madame de Lorimier assiste au procès de son mari qui dure du 11 au 21 janvier 1839. Durant 10 jours elle voit son mari en butte à la mauvaise volonté des juges, à la vengeance des témoins. Un peu plus tard Henriette Chevalier de Lorimier apprend que son mari montera sur l'échafaud le 15 février 1839. F.-X. Prieur a écrit d'elle:

...De toutes les pauvres femmes des condamnés, madame

De Lorimier est celle qui m'inspirait le plus de pitié c'était comme un pressentiment, et puis cette pauvre famille, en perdant son chef, perdait tout moyen d'existence. Notre malheureux ami parlait chaque fois à sa femme de sa pauvreté, il cherchait à trouver quelque moyen à lui suggérer pour

27. Louis-Joseph-Amédée Papineau, "Journal d'un Fils de la Liberté", 3; 464. 28. L.-O. David, Les Patriotes 1857-1838. (Montréal, 1925), 205. 
pouvoir élever ses enfants et, dans l'impossibilité de trouver ce moyen sous des formes saisissables, il finissait toujours par lui dire: "La divine Providence ne t'abandonnera pas"29.

[ Le 14 février] Vers les trois heures de l'après-midi madame de Lorimier, accompagnée de la sœur et de la cousine de son mari, et conduite par un M. de Lorimier, cousin du condamné, entrèrent dans notre logement. Madame de Lorimier portait sur sa figure une expression de douleur à fendre le cœur, mais elle ne pleurait pas: ses deux compagnes fondaient en larmes.

Nous avions pris des arrangements pour donner à nos deux malheureux amis un dîner d'adieu. La table chargée de mets préparés sur notre ordre par le geôlier, avait été placée dans une pièce située près de la porte et qui donnait sur le corridor. A quatre heures on se mit à table, Hindenlang présidait au banquet. De Lorimier n'occupa pas le siège qui lui était réservé; mais il vint prendre avec nous un verre de vin. Pendant le repas, il se promenait dans le corridor, ayant madame de Lorimier au bras; les autres membres de sa famille occupaient des sièges, tantôt dans sa cellule, tantôt dans le corridor: les dames, de temps à autre, prodiguaient à la malheureuse épouse des caresses de consolation... A dix heures le geôlier vint nous dire qu'il fallait entrer. C'était le moment que ce pauvre de Lorimier redoutait tant, et que, nous aussi, nous voyions venir avec un déchirement de cœur. Quelques parents et amis étaient venus s'ajouter aux trois personnes de la famille qui accompagnaient madame de Lorimier et qui devaient être chargées de la pénible, mais charitable mission, de la reconduire en ville.

La pauvre jeune femme allait donc dire à son mari un éternel adieu! A la suite de bien des hésitations, des sanglots et des larmes, elle se jeta à son col et s'évanouit de nouveau. De Lorimier la souleva dans ses bras, et la tenant comme un enfant qu'on va déposer dans son berceau, il se dirigea vers la porte, les yeux attachés sur cette figure agonisante de la compagne de sa vie: arrivé sur le seuil, il dépósa un baiser sur le front décoloré de sa femme, et la remit entre les bras de ses parents, en leur recommandant d'en avoir tous les soins possibles... et la porte se referma sur nous"30.

Puis De Lorimier passe une partie de la nuit à écrire des lettres. Il faut retenir celle qu'il écrit le matin même de son exécution, à sa 61-62.

29. F.-X. Prieur, Notes d'un Condamné Politique de 1898 (Montréal, 1884),

30. Ibid., 67-70. 
femme, et trouvée sur son cœur après sa mort. Cette lettre révèle une part des tortures endurées par une Canadienne à l'époque et de quel appui elle fut pour son mari.

Dans le peu de temps qui s'est écoulé depuis le jour de notre union sacré jusqu'à ce jour, tu m'as rendu, ma chère femme, vraiment heureux. Ta conduite envers moi a toujours été irréprochable et dictée par l'amitié, la bonté et la sincérité.

J'ai toujours su apprécier tes vertus. Aujourd'hui même des hommes altérés de sang, m'arrachent à tes bras; mais ils ne réussiront jamais à effacer mon souvenir de ton cœur, j'en sưis convaincu. Ils t'enlèvent ton appui et ton protecteur et le père de tes chers jeunes petits enfants. La Providence et des amis de mon pays en auront soin. Ils ne m'ont même pas donné le temps de voir mes chères petites filles, de les presser sur mon cœur et de leur dire un éternel adieu. Quant à toi, ma chère, tu dois prendre courage et te pénétrer de la pensée qu'il faut que tu vives pour l'amour de tes enfants infortunés qui auront grandement besoin des soins d'une mère tendre et dévouée. Je t'assure, ma chère Henriette, que si des régions cólestes, il m'est permis de veiller sur toi et de t'aider, je fortifierai ton cœur brisé. Je ne te verrai plus dans ce monde. Je finis ma chère Henriette, en offrant à Dieu les prières les plus sincères pour ton bonheur. Hier soir, tu as reçu mon dernier adieu. Ton tendre et affectueux mari enchaîné comme un meurtrier dont les mains seront liées, te souhaite, ma chère Henriette, de jouir de tout le bonheur dont ton cour brisé sera susceptible à l'avenir. Sois donc heureuse, ma chère et pauvre femme, ainsi que mes chers petits enfants, c'est le vœu le plus ardent de mon âme. Adieu, ma tendre femme, encore une fois adieu; vis et sois heureuse.

Ton malheureux mari,

Chevalier de Lorimier $^{31}$.

Nous ne prétendons pas avoir établi en son entier le rôle de la femme pendant l'insurrection. Puisse notre modeste travail susciter d'autres recherches.

Madame Marcelle Reeves-Morache

31. Nous empruntons le texte de cette lettre à L.-O. David, Les Patriotes de 1837-1838 (Montréal, 1913), 249-250. Nous n'avons pu en retracer l'original. S'il existe quelque part, on serait bien aimable de nous le faire savoir. 